\title{
More persistent bacterial than fungal associations in the microbiota of a pest
}

3 Groningen Institute for Evolutionary Life Sciences, University of Groningen, P.O. box 11103, 9700 CC,

Author information details:

1. Kiran Gurung

corresponding author:k.gurung@rug.nl

9 Groningen Institute for Evolutionary Life Sciences, University of Groningen, P.O. box 11103, 9700 CC,

10 Groningen, The Netherlands

11 2. Stefanie N. Vink

12 Groningen Institute for Evolutionary Life Sciences, University of Groningen, P.O. box 11103, 9700 CC,

13 Groningen, The Netherlands

14 3. Joana Falcão Salles

15 Groningen Institute for Evolutionary Life Sciences, University of Groningen, P.O. box 11103, 9700 CC,

16 Groningen, The Netherlands

\section{Bregje Wertheim}

18 Groningen Institute for Evolutionary Life Sciences, University of Groningen, P.O. box 11103, 9700 CC,

19 Groningen, The Netherlands

20

21

22 


\section{Abstract}

26 The invasive fly Drosophila suzukii is a pest that can infest a diverse range of intact, ripening

27 fruits, using its serrated ovipositor. This constitutes a different niche compared to the rotting

28 fruits its ancestors use, especially because these intact fruits have limited quantities of microbes

29 and soluble nutrients for the developing larvae. To investigate the potential role of microbial

30 associations in the niche expansion of this invasive fly, we characterized the bacterial and

31 fungal communities of $D$. suzukii and various wild fruits from which they developed. To assess

32 cross-generational microbial associations, we also lab-reared fly populations and characterized

33 their microbial communities. Diversity metrics of microbial communities differed significantly

34 between flies and fruits. Different fruit types varied substantially in microbial composition, while

35 flies showed relatively uniform bacterial communities, irrespective of the fruit source they

36 developed on. After lab-rearing, bacterial communities still showed considerable overlap with

37 those of wild flies. Fungal communities of flies and fruits showed larger resemblance, with a

38 substantial overlap between wild flies and the corresponding fruits on which they had

39 developed. Our study thus reports that the fungal community structure in these pests largely

40 reflects those on the breeding substrates, while these flies might have formed more persistent

41 associations with bacteria and transmit these across generations rather than obtaining them

42 from their food source.

43 Keywords: bacterial community, fungal community, microbiota, core microbes, drosophila,

44 invasive insect, insect microbiota 


\section{Introduction}

50 The microbial communities associated with insects are highly diverse, and the dynamics within

51 and between members of the microbiome can affect the fitness and behavior of insects in

52 various ways (Gurung et al., 2019). Studies on insect microbiota have demonstrated that

53 various factors shape the microbial community composition, ranging from life stages,

54 environment, host genetics and diet (Yao et al., 2019). In some cases, microbes also contribute

55 towards the pest status of invasive insects (Lu et al., 2016). When pest insects are (at least

56 partially) dependent on microbes for their performance or fitness, these microbes have the

57 potential of being utilized as pest management tools, and often one of the first steps is examining the microbiota profiles and their associations in the pests (Crotti et al., 2012; Lewis et

A pest of ripening fruits, Drosophila suzukii has its origin in Asia (Drosophila suzukii, CABI.

612019 ), and has successfully invaded several parts of America and Europe in the last decade

62 (Calabria et al., 2012; Cini et al., 2014). The female lays eggs on intact ripening fruits and

63 damages them, which is often accompanied by fruit tissue collapse, due to both feeding of the

64 developing larvae inside the fruit, and the rot that is frequently induced by the infestation with $D$.

65 suzukii (loriatti et al., 2018). Fruits become unsuitable for sale, resulting in huge economic

66 losses for fruit growers (Goodhue et al., 2011; Atallah et al., 2014). Drosophila suzukii infests a

67 large range of soft fruits, cherries, and different types of berries (Walsh et al., 2011; Rota-

68 Stabelli et al., 2013). The ability of $D$. suzukii to colonize intact fruits is driven by its serrated

69 ovipositor, which allows it to puncture the skin of fruits. This is a morphological innovation that 
70 enabled the species to expand from its ancestral niche of rotting and decomposing fruit to also

71 infest fresh and ripening fruits (Atallah et al., 2014).

72 Ripening fruits have fewer nutrients (proteins and sugars) (Silva-Soares et al., 2017) and also

73 harbor a more limited number of microbes than fermenting fruits. This poses possible dietary

74 challenges to the larvae of $D$. suzukii that develop mostly inside the fruit host. A number of

75 Drosophila species depend on yeasts during their larval stage for essential nutrients, and feed

76 on these yeasts when they are developing on fruits (Starmer \& Fogleman, 1986; Carvalho et al,

77 2010). In line with this, earlier research demonstrated that germ-free $D$. suzukii larvae failed to

78 develop on protein-poor and fruit-based artificial diets, but this could be rescued with the

79 supplementation of microbiota (Bing et al., 2018). If the microbial communities on intact fruits

80 are indeed insufficient to sustain larval development, then during oviposition $D$. suzukii females

81 may possibly inoculate the fruits with a set of microbes to supply their developing offspring with

82 the required microbiota. Association with some microbes may thus have an essential impact on

83 the life history of these flies. Furthermore, microbes vectored by the flies may have plant

84 pathogenic potential, causing the fruit collapse (Hamby and Swett, 2015). The question as to

85 what kind of microbial associations these pests might have can thus provide us with a better

86 understanding of microbial contribution in the niche expansion of this invasive pest, in its broad

87 life history, as well as in its pest status.

88 One of the earliest studies on the bacterial community composition in field-caught $D$. suzukii

89 revealed Tatumella to be a dominant bacterium across larval and adult stages (Chandler et al.,

90 2014). Although their exact role on insect host fitness is not yet known, the species T. ptyseos

91 reportedly showed plant pathogenic traits (Marín $\square$ Cevada $\vee$ et al., 2010). Furthermore, bacteria

92 belonging to the families of Acetobacteriaceae, Enterobacteriaceae and Firmicutes have also

93 been reported in D. suzukii. Some of these bacteria are associated with Drosophila in general

94 and provide fitness benefits (Bing et al., 2018). Also yeasts such as Hanseniasporum, Pichia, 
95 and Issatchenkia, impact various life history traits of D. suzukii (Lewis and Hamby, 2019). Such

96 reports on various microbial associations, point out a question whether the pest associates with

97 microbes through exposure on their breeding site, or whether they might be retaining and

98 transmitting microbiota in a more persistent association.

99 To investigate the microbial associations of this invasive fly, we characterized the similarities

100 and differences among the bacterial and fungal communities of $D$. suzukii and various wild fruits

101 from which they developed. The microbial communities that are typically associated with

102 surfaces of various fruits and vegetables can vary considerably (Leff and Fierer, 2013). When

103 developing larvae rely mostly on the microbial communities that they encounter on these fruits,

104 we would expect that emerging flies from different fruits would exhibit high variations in their

105 community patterns and align with those of the corresponding fruits. Alternatively, when $D$.

106 suzukii formed more persistent associations with some microbes that they inoculate into the

107 fruits while ovipositing, we would expect flies emerging from different fruit types to harbour

108 similar microbiota. To explore whether emerged flies exhibit consistent microbial associations

109 across various fruits, or whether fruit source plays an important role in determining the flies'

110 microbiome, we sampled the microbiome composition (bacteria and fungi) of fruits and the

111 corresponding emerging D. suzukii flies, collected at four different locations in the Netherlands.

112 Additionally, to identify members that potentially have a more intimate relationship with $D$.

113 suzukii, and are vertically or horizontally transmitted, we subjected some of these wild flies to

114 lab-rearing conditions and evaluated the changes in their microbiome after several generations.

\section{Methods}

\section{Sampling wild flies and fruits}


118 We collected fruits that were infested with D. suzukii in The Netherlands in 2018, across four

119 different locations (between 7.5 - $200 \mathrm{~km}$ apart) and five fruit types (Summary in table 1). In

120 three of these locations, we sampled multiple different fruit types or cultivars. Infestation was

121 determined by identifying the dented spots on the fruits that resulted from egg laying. A large

122 number of collected fruits were individually placed in plastic cups, parafilm-sealed and brought

123 into the lab to further incubate them in bottles with autoclaved sawdust (saw dust soaks up

124 unwanted moisture from decomposing fruits) until the adult flies emerged. Incubation conditions

125 were $20^{\circ} \mathrm{C}, 65 \%$ relative humidity and $16 \mathrm{hr}: 8 \mathrm{hr}$ light:dark cycle. The emerging flies (referred

126 as "wild flies") from each fruit and the corresponding fruits were collected and stored at $-80^{\circ} \mathrm{C}$

127 until DNA extraction. An additional batch of flies that emerged from the same cherry, strawberry

128 or elderberry fruits was subsequently lab-reared (referred as "lab-reared flies").

\section{Lab rearing}

130 We started three lab cultures from wild flies that emerged from 1) cherry collected in Randwijk,

131 2) strawberry collected in Dirksland, and 3) elderberry collected in Hoogeveen. These strains

132 were reared in the lab on artificial food (see supplementary file for the fly food composition), for

133 approximately 10 generations, before being collected and stored at $-80 \square \mathrm{C}$ for microbiome

134 characterization. The fly food contained sucrose, glucose, agar, cornmeal, wheatgerm, soy

135 flour, molasses, yeast, ethanol, and was supplemented with antimicrobial agents (propionic acid

136 and nipagin) to reduce mold contamination. The founding population of flies was composed of

137 8-10 individuals per fruit type. Each successive generation, at least fifty individual flies were 138 transferred to the fresh medium. 
141 We collected five biological replicate samples for each fruit type: Each fly sample consisted of

142 five female flies (wild and lab-reared flies) that emerged from a single fruit, and approximately

$1431 \mathrm{~g}$ of fruit was excised with sterilized bladed for the corresponding fruit samples. DNA

144 extractions were performed using Qiagen Soil DNA extraction kit according to manufacturer's

145 protocol. Prior to extraction, pooled flies in $2 \mathrm{~mL}$ centrifugation tubes were washed once in sterile

146 water by vortexing for approximately 10 seconds to remove saw dust. Finally, in order to check

147 for any kit-associated contamination (Glassing et al., 2016), five extraction controls with no

148 samples were subjected to DNA extraction and further sequencing.

149 Following extractions, we quantified DNA using NanoDrop2000 (Thermo Fisher Scientific, MA, 150 USA). Sequencing was outsourced to the University of Minnesota Genomics Center (USA), on

151 Illumina MiSeq platform (V3 Chemistry and 2×300 paired end run), targeting the V4-V6 region of

152 the 16S rRNA gene and ITS1 region for bacteria and fungi respectively (Primer sequence

153 information in supplementary file).

\section{Sequence analyses}

155 We processed the sequences in QIIME2 platform (version 2019.10, Boylen et al., 2019). To

156 avoid losing a substantial amount of reads after pairing (due to non-overlapping coverage of the

157 amplicon), we chose to analyze only the forward reads for both bacteria and fungi. For bacteria,

158 we trimmed the primers using the 'Cutadapt' plugin (Martin, 2011). We denoised and chimera

159 filtered the sequences using DADA2 plugin with its default settings at phred score of at least 25

160 and truncation length of $220 \mathrm{bp}$ (Callahan et al., 2016). Taxonomic assignment was done using

161 RDP classifier (Wang et al., 2007). We removed sequences belonging to archaea, eukaryotes,

162 chloroplast and mitochondria. For fungi, we used ITSxpress plugin (version 1.7.2, Rivers et al.,

163 2018) to extract the fungal ITS1 region. Denoising and chimera filtering was performed using

164 DADA2 with a truncation length of zero. Taxonomic assignment was performed using the 
165 UNITE database (version 8_99_04.02.2020, Abarenkov et al., 2010) that was trained and

166 classified. We used the feature table and taxonomy table for both bacteria and fungi and

167 additionally phylogenetic tree for bacteria generated from the QIIME2 workflow in R (version

168 3.6.3, $\mathrm{R}$ Core Team). The reads in the extraction controls were subtracted and removed from

169 the read abundances in the samples prior to downstream analysis (taxa found in controls are

170 provided in the supplementary file).

\section{Statistical analysis}

172 After excluding controls, there were a total of 105 samples that comprised fruits, wild flies and

173 lab-reared flies. This whole dataset was used to provide an overview of the microbial

174 communities associated with flies and fruits. Comparative analyses were done on subsets: for

175 the fruit-wild fly comparisons (90 samples, bacterial and fungal community) and for the lab-

176 reared fly-wild fly comparisons (30 samples, bacterial community). We did not include the

177 analyses from fungal communities of lab-reared and wild fly subsets as we used antifungal

178 agents which could be a highly confounding factor in their community pattern, but we have

179 added the data in the supplementary file.

180 For the fruit-wild fly comparisons, the data were rarefied to a sequencing depth of 4500 for

181 bacteria and 7800 for fungi, resulting in the loss of seven and six samples for bacterial and

182 fungal communities respectively. For the lab-reared fly-wild fly comparison, the reads were

183 rarefied to a sequencing depth of 4500 for bacteria with no loss of samples. Analysis on alpha

184 and beta diversity were performed on rarefied data, while remaining analyses were performed

185 on unrarefied data.

186 For bacterial alpha diversity we calculated richness (observed ASVs) and Faith's phylogenetic

187 diversity using packages phyloseq (version 1.30.0, McMurdie and Holmes, 2013) and picante

188 (version 1.8.1, Kembel et al., 2010). Fungal alpha diversity was calculated based on richness 
and Shannon index using phyloseq. We used the Kruskal-Wallis test (Kruskal and Wallis, 1952)

190 for comparing the alpha diversity between different sample types (i.e., the different types of fruit

191 sources, and the different groups of emerging wild flies), followed by post-hoc Dunn tests with

192 Benjamini-Hochberg p-adjustment method (Dunn, 1961; Benjamini and Hochberg, 1995) using

193 phyloseq and dunn.test (version 1.3.5).

194 We determined the beta diversity by PCoA based on UniFrac distance metrics for bacterial 195 communities (Lozupone and Knight, 2005) and Bray Curtis metrics for fungal communities 196 (Beals, 1984) using the packages phyloseq and vegan (2.5-6, Oksanen, 2015). We tested for 197 statistically significant differences among all the fruits-wild flies, and between lab-reared flies198 wild flies using PERMANOVA (Anderson, 2014). Differences in community dispersion was 199 checked by PERMDISP (Anderson, 2006) for the significant p-values resulting from 200 PERMANOVA.

201 Using procrustes analysis, we further tested the degree of association of the bacterial and 202 fungal communities based on the Bray Curtis metrics, between the fruits-wild fly subset as well 203 as between the wild and lab-reared fly subset (Jackson, 1995), with codes adapted from van 204 Veelen et al. (2020).

205 We assessed taxonomic relative abundances across fruits-wild flies and in the lab-reared flies206 wild flies using package microbiome (version 1.12.0, Lahti and Shetty, 2017), with default 207 parameters and transformation set to 'compositional'. 
211 The alpha diversity of bacterial communities across the fruits and wild flies differed by fruit types

212 in terms of observed ASVs $\left(X^{2}=25.829\right.$, p-value $<0.001$, Figure 1a) and Faith's phylogenetic

213 diversity $\left(X^{2}=12.085, \mathrm{p}\right.$-value $<0.001$, Fig.S1a). In general, alpha diversity was higher in wild

214 flies than in fruits, except for the flies originating from raspberries in both locations Winssen and

215 Dirksland (dunn test, p-value=0.05).

216 Richness and Shannon diversity index of fungal communities also differed significantly in fruits

217 and wild flies according to the fruit types $\left(X^{2}=22.702, p\right.$-value $<0.001$, Fig $1 b ; X^{2}=48.429$, $p$ -

218 value $<0.001$, Fig. $S 1 b$; dunn test, $p$-value=0.05), but contrary to richness for bacterial

219 communities, the fungal communities on fruits generally showed higher alpha diversity than the

220 flies.

222 Microbial community patterns in fruits and associated flies

223 To assess whether the microbiota of the flies would reflect those of the fruits, we compared the 224 microbiome composition of the fruit sources and wild flies (Figure 2). The bacterial communities 225 of wild flies clustered together and away from their corresponding fruit sources, thereby 226 indicating a strong separation across these two sample types. For bacterial communities, 227 PERMANOVA based on weighted UniFrac distance revealed significant differences between 228 the wild fly and their corresponding fruits $\left(R^{2}=0.2688\right.$, $p$-value=0.001, figure $\left.2 a \& 2 b\right)$. 229 Additionally, the communities were homogeneous in their dispersion (PERMDISP, p230 value $=0.755)$. Based on unweighted UniFrac, PERMANOVA $\left(R^{2}=0.115, p\right.$-value $=0.001$, figure 231 S2) showed significant difference and dispersion was not homogenous (PERMDISP, $p$-value = 2320.001 , figure S2). While replicate samples for the same fruit type per location clustered mostly 233 together, they showed some variance both within and among fruit types (figure S2). 
234 The beta diversity of fungal communities, assessed using PERMANOVA on Bray-Curtis

235 distance, also showed differences between wild flies and their corresponding fruits $\left(R^{2}=0.022\right.$,

$236 \mathrm{p}$-value=0.021, figure $2 \mathrm{c} \& 2 \mathrm{~d}$ ). Unlike the bacterial communities, however, the fungal

237 communities showed no clear clustering into either fruit or fly samples (Figure 2d); rather the

238 fruit and fly samples overlapped considerably within fruit type, but fruit types differed

239 substantially from each other. The dispersion was heterogeneous (PERMDISP, F-value=8.30,

240 -value=0.005), revealing differences in within-group variances. We observed considerable

241 overlap of the fungal communities between the wild fly and its corresponding fruit source, as

242 shown from the clustering by fruit source in most of the combinations (Figure $2 \mathrm{~d}$ ). Additionally,

243 considerable clustering occurred between different fruits and flies from similar locations (as

244 noted for the three cherry cultivars from Randwijk, and among strawberries, raspberries and

245 blackberries from Dirksland).

246 We further performed procrustes analysis to assess if the microbial communities across sample

247 subsets covaried (Table 2). For the bacterial communities, we did not observe concordance

248 between the wild flies and the corresponding fruit subset $\left(\mathrm{M}^{2}=0.934, \mathrm{p}\right.$-value $\left.=0.19\right)$. In contrast,

249 association between fungal communities of wild flies and corresponding fruits was significant

$250 \quad\left(\mathrm{M}^{2}=0.554, \mathrm{p}\right.$-value=0.001).

\section{Bacterial community across wild and lab-reared flies}

253 Bacterial richness in lab-reared flies was similar to that of their wild counterparts, except for the

254 samples from strawberry, where lab-reared flies showed a higher alpha diversity (figure 3a).

255 Overall, we did not find any difference between the wild and the lab flies ( $p$-value=0.40). Also,

256 the Faith's phylogenetic diversity indices did not differ significantly across the sample types (p-

257 value $=0.101$, figure S3). 
258 We observed significant differences in beta diversity of the bacterial communities between lab-

259 reared flies and their respective wild flies in composition $\left(R^{2}=0.06, p\right.$-value $=0.011$, figure $\left.3 b\right)$, but

260 only $6 \%$ of the variation could be explained by the rearing environment. We did not observe any

261 differences in the dispersion (PERMDISP, F-value=3.15, p-value=0.66). Further, the

262 correspondence in bacterial communities between wild flies and lab-reared flies was significant

$263\left(\mathrm{M}^{2}=0.5298, \mathrm{p}\right.$-value=0.003, Table 2).

\section{Community composition and core microbes among different sample types}

266 We profiled the relative abundances of top-10 most abundant bacteria and fungi across the

267 different samples (Figure 4). At the genera level, we observed Candidatus Carsonella, Ca.

268 Scalindua, Gluconobacter, Minicystis, Omnitrophica genera incertae sedis, Pantoea, Pelospora,

269 Stella, Tatumella, Tephidisphaera among the top 10 abundant bacterial genera across the wild

270 flies and fruit samples. Some of these genera were limited to only the flies, or were recorded at

271 a higher abundance in flies than in the corresponding fruits (Figure 4a). We further identified

272 four bacteria that were found in at least $95 \%$ of wild fly samples at an abundance threshold of

2730.001 (Figure 4b). These were Succinatimonas, Pelospora, Ca. Carsonella and Ca.

274 Omnitrophica. In the fruit samples, we were unable to identify any bacterial genera that were

275 common to all the fruit samples, even at lower prevalence. Furthermore, these fly core bacteria

276 were only retrieved from a few fruit samples and in much lower abundances than in wild flies,

277 except for one fruit sample (figure S4). Three of these core bacteria were also observed in the

278 lab-reared flies (Figure 4C). In addition, we found Acetobacter in the lab-reared flies, which was

279 missing in most of the ancestral wild flies emerging from cherries and elderberries.

280 Both yeasts and molds made up the fungal composition across the fruit and wild fly subsets,

281 mostly belonging to the genera Aureobasidium, Saturnispora, Botrytis, Candida, Cladosporium, 
282 Saccharomycopsis, Gibberella, Metschnikowia, Penicillium, and Aspergillus, with the latter three 283 also being present in the lab-reared flies (Figure 4d and S5c). We did not find any core fungal

284 ASVs at 95\% prevalence across either fruits or wild flies, or across both combined.

285 Furthermore, there was no core fungal ASV common in the wild fly subset even with relaxed

286 criteria of lower prevalence and detection threshold. In the fruit samples, however, we did find 287 Mycosphaerella tassiana at a prevalence of $70 \%$ and 0.0001 abundance threshold. Hence, 288 almost all wild flies shared a subset of bacteria, and occurrence of some of these bacteria was 289 rare in fruits. In contrast, fungi were not commonly shared among the wild flies.

\section{Discussion}

293 In this study we characterized the bacterial and fungal communities associated with wild $D$. 294 suzukii flies, an invasive pest insect originating from Asia, which causes severe damage in the 295 production of soft fruits around the globe (Walsh et al., 2011). The success of this species lies in 296 its ability to perforate the fruit skin to lay eggs on ripening fruits, as well as its capacity to 297 develop in a large variety of these fruits. This new niche of ripening fruits may lack essential 298 nutrients that microbes could be providing (Bing et al., 2018). Our primary focus was to study 299 the microbiome in D. suzukii, as well as its associated fruit substrates, to assess whether the 300 flies acquired their associated microbiota mostly from exposure to the microbial communities of 301 the fruits on which they developed - in this case, we expected substantial overlap of the fruit 302 and fly microbiota with differences in microbial composition for flies emerging from different fruit 303 types - or whether they have formed more persistent associations with some microbiota that 304 they retain irrespective of their fruit hosts. 
305 We observed significant differences in bacterial and fungal diversity among different fruit types,

306 but the flies that emerged from these fruits had a remarkably uniform bacterial community

307 composition. In contrast, the fungal community distribution in the flies largely resembled those in

308 their host fruits. Flies that were lab-reared for 10 generations still harboured some of the

309 bacterial genera that largely resembled those of their wild ancestors. We observed no significant

310 difference in alpha diversity metrics between bacterial communities of lab-reared and wild flies.

311 Perhaps this could also indicate that lab-rearing for 10 generations might not have been

312 sufficient to change the community pattern. For fungal communities, although we observed no

313 significant difference in beta diversity between the lab-reared and wild flies, we did see a severe

314 reduction in alpha diversity for lab-reared flies, but this is likely attributable to the use of anti-

315 fungal agents (i.e., proprionic acid and nipagin) in the artificial diet (Figure S5a and S5b). Thus,

316 the bacterial communities in emerging flies were largely shaped irrespective of the fruit type and

317 are distinct from the bacterial communities on the fruits itself. In contrast, the fruit source most

318 likely potentiated the fungal community distribution in the flies. At the level of ASVs, several of

319 the microbes present in the wild flies also seem to be present in the lab-reared samples, 320 suggesting that they were vertically or horizontally transmitted.

322 It is important to notice that in our study we did not analyze the larval microbiome, as we were 323 interested specifically in the associated microbes of the adults. In holometabolous insects like 324 Drosophila, metamorphosis induces changes at morphological and physiological level, which 325 restructures several tissues and might also result in shedding of microbes (Tissot and Stocker, 326 2000; Johnston and Rolff, 2015). However, sometimes, depending on the family or life history of 327 the insects, a few selected microbes might be retained (Johnston and Rolff, 2015). In order to 328 verify whether such restructuring during development results in microbial shifts for $D$. suzukii, 329 future studies should also focus on determining the microbiome of wild $D$. suzukii larvae as well 
as pupae collected from fruits across a broader geographical range. In line with our findings, another study on associated microbes of the Bactrocera tephritid fruit flies showed difference in bacterial community pattern for fruits and larvae (Majumder et al., 2019). In Drosophila species, Martinson et al. (2017) reported the bacterial community composition in flies to be distinct from

334 their source food. Although we do not yet know how this separation of fruit and fly bacterial communities can persist, the continuation of the bacterial associations for 10 generations on artificial diet suggest that perhaps ovipositing females can transmit bacteria across generations to their offspring. And in addition to diet, host type may also have been a factor driving the community composition in our fly samples.

Drosophila suzukii flies spend a substantial amount of time in close association with the fruits

341 from which they emerge as adults. We therefore expected to observe a subset of microbes commonly shared across both fruits and flies. At the genera level, we observed Gluconobacter,

343 Pantoea, Tatumella across the fruits and some of the wild flies, as reported by previous studies

344 (Chandler et al., 2014; Vacchini et al., 2017; Solomon et al., 2019). We also observed bacteria

345 Ca. Carsonella, Ca. Scalindua, Succinatimonas etc. to be more abundant in the flies, and

346 Acetobacter to be also present in the lab flies. Some of the prevalent fungal members of the 347 genera Penicillium and Metschnikowia were common among wild flies and fruits, with the former 348 being dominant in the lab-reared flies as well. In addition, few other fungi like Pichia, Botrytis, 349 Cladosporium were also noted in some of the fly samples. However, no fungal genera were 350 consistently shared among wild flies or fruits. The fungal community composition varied among 351 fruit types, and those of the emerged flies showed substantial overlap. Similar to our findings, 352 previous studies on coleopteran insects also suggested a strong role of the immediate 353 environment in structuring the fungal communities in the insects (Kudo et al., 2019; Rassati et 354 al., 2019). 
355 Understanding the interactions between microbiome and insects is interesting from an 356 evolutionary perspective, as it may facilitate niche expansions and adaptations to challenging 357 environmental conditions. Furthermore, the core bacteria that we observed in our samples 358 reportedly engage in carbohydrate and amino acid metabolic pathways (Riley et al., 2017; 359 Morotomi et al., 2010; Stackebrandt and Hespell, 2006; Matthies et al., 2000). In the case of 360 pest insects, it is also highly relevant to understand what determines the composition of these 361 microbial communities, as this may reveal unknown aspects on their fundamental biology, 362 sometimes also providing insight into vulnerabilities that can be exploited in pest management 363 strategies (Raza et al., 2020). Culture based and sequencing approaches have revealed the 364 influence of environmental factors on the bacterial community composition of pest insects, such 365 as diet, climate and geographic location (Rizzi et al., 2013; Jones et al., 2019; Colman et al., 366 2012). To understand the processes by which associations between pest insects and microbiota 367 arise, it is important to investigate the microbial composition on their food source (Behar and 368 Jurkevitch, 2008; Beaulieu et al., 2017), which has been reported as a potential source from 369 which insects can acquire their associated microbiota (Jones et al., 2019). Yet, it is also 370 important to realize that specific insect-microbe associations can arise that transcend the 371 intermediate of a food source.

372 The ability of $D$. suzukii to grow on such a wide range of fruits makes it an interesting system to 373 explore whether fruit source plays an important role in determining its microbiome. Microbial 374 communities vary considerably among various fruits and vegetables (Leff and Fierer, 2013), as 375 we corroborated in the analyses of microbial communities on our fruit samples. We speculated 376 that flies developing on different fruits would exhibit high variations in their community patterns 377 and align with those of the corresponding fruits when they relied on their fruit hosts for acquiring 378 microbes. Our results, however, showed that the bacterial communities of the wild flies were 379 largely distinct from those of the fruits, and showed large similarities irrespective of fruit type. 
380 Some of the microbial members that were found in wild flies from different fruits were also

381 retained after 10 generations of lab-rearing. Based on these findings, we propose that $D$.

382 suzukii may have formed persistent associations with some of these bacteria. In order to

383 investigate what precisely fosters the host-microbiome interactions in this pest, studies similar to

384 Ben-Yosef et al. (2014) could likely answer whether indeed there is any particular microbial

385 associations that drives the pest status of $D$. suzukii. The fungal communities seemed to be less

386 persistent, and here we did see more alignment in community patterns between fruits and

387 emerging flies. This does not necessarily imply that these fungi are unimportant in the niche

388 expansion, as they could be providing essential nutrients, e.g., sterols (Carvalho et al, 2010).

389 However, it does appear that the associations with fungi are more transient than those with

390 bacteria.

391 Concluding, our study reports the microbial community structure of Drosophila suzukii across a

392 range of fruits. By characterizing the microbial communities of both the flies and the fruits from

393 which they emerged, we could assess the similarity and differences between these communities

394 across the fruits and the flies, in several different fruit sources. The characterization of these

395 microbial communities represented, thus, an important step to identify any potential symbiotic

396 relationship between these flies and bacterial or fungal species 
Acknowledgments: We thank Herman Helsen who provided the details of infestation and the growers. We are grateful to Tom Groot (Dirksland), Maria Buitenkamp and Edo Biewinga

411 (Hoogeveen); Gijs Gerritse (Randwijk); Jeroen Spitzen (Winssen) who provided access to their 412 garden and orchards. This work was supported by the Adaptive Life scholarship program (2017) 413 awarded to KG by the University of Groningen. We thank the Center for Information Technology 414 of the University of Groningen for their support and for providing access to the Peregrine high 415 performance computing cluster. This research has been carried out in the groups of 416 Evolutionary Genetics and Microbial Ecology at the Groningen Institute for Evolutionary Life 417 Sciences (GELIFES) according to the requirements of the Graduate School of Science and 418 Engineering (Faculty of Science and Engineering, University of Groningen; Groningen, the 419 Netherlands).

420 Author contribution: KG, JFS, BW conceived the experimental plan. KG performed all 421 analyses. SNV and JFS provided support with sequence analysis. BW provided support with 422 statistical analysis. KG wrote the original draft. KG, SNV, JFS and BW reviewed and edited 423 subsequent drafts.

424 Competing interest: The authors declare no competing financial (or any other) interests.

\section{$425 \quad$ References}

426 - Abarenkov, Kessy, et al. The UNITE database for molecular identification of fungi recent updates and future perspectives. New Phytol 2010; 186.2: 281-285. 
- Anderson MJ. Permutational multivariate analysis of variance (PERMANOVA). Wiley statsref: statistics reference online. $2014 ; 14: 1-5$

- Atallah J, Teixeira L, Salazar R, Zaragoza G, Kopp A. The making of a pest: the evolution of a fruit-penetrating ovipositor in Drosophila suzukii and related species. Proc R Soc Lond [Biol]. 2014; 281(1781):20132840.

- Bauer DF. Constructing confidence sets using rank statistics. J Am Stat Assoc. 1972; 67(339):687-90.

- Beals EW. Bray-Curtis ordination: an effective strategy for analysis of multivariate ecological data. Adv Ecol Res. 1984; 14:1-55.

- Beaulieu M, Franke K, Fischer K. Feeding on ripening and over-ripening fruit: interactions between sugar, ethanol and polyphenol contents in a tropical butterfly. J Exp Biol. 2017; 220(17):3127-34.

- Behar A, Jurkevitch E, Yuval B. Bringing back the fruit into fruit fly-bacteria interactions. Mol Ecol. 2008; 17(5):1375-86.

- Benjamini Y, Hochberg Y. Controlling the false discovery rate: a practical and powerful approach to multiple testing. J R Stat Soc Series B Stat Methodol. 1995; 57(1):289-300. larvae to overcome host defences. R. Soc. Open Sci. 2015; 2(7):150170.

450 - Bolyen E, Rideout JR, Dillon MR, Bokulich NA, Abnet CC, Al-Ghalith GA, Alexander H, extensible microbiome data science using QIIME 2. Nat biotechnol. 2019; 37(8):852-7. 
- Calabria G, Máca J, Bächli G, Serra L, Pascual M. First records of the potential pest species Drosophila suzukii (Diptera: Drosophilidae) in Europe. J Appl Entomol. $2012 ; 136(1-2): 139-47$.

- Callahan BJ, McMurdie PJ, Rosen MJ, Han AW, Johnson AJ, Holmes SP. DADA2: highresolution sample inference from Illumina amplicon data. Nat Methods. 2016; 13(7):5813.

- Carvalho M, Schwudke D, Sampaio JL, Palm W, Riezman I, Dey G, Gupta GD, Mayor S, Riezman H, Shevchenko A, Kurzchalia TV. Survival strategies of a sterol auxotroph. Development. 2010;137(21):3675-85.

- Chandler JA, James PM, Jospin G, Lang JM. The bacterial communities of Drosophila suzukii collected from undamaged cherries. PeerJ. 2014; 2:e474.

- Cini A, Anfora G, Escudero-Colomar LA, Grassi A, Santosuosso U, Seljak G, Papini A. Tracking the invasion of the alien fruit pest Drosophila suzukii in Europe. J Pest Sci. 2014; 87(4):559-66.

- Colman DR, Toolson EC, Takacs-Vesbach CD. Do diet and taxonomy influence insect gut bacterial communities? Mol Ecol. 2012; $21(20): 5124-37$.

- Crotti, E., Balloi, A., Hamdi, C., Sansonno, L., Marzorati, M., Gonella, E., Favia, G., resource for the management of insect-related problems. Microb Biotechnol., 5(3), 307-

473 - Currie CR. A community of ants, fungi, and bacteria: a multilateral approach to studying $474 \quad$ symbiosis. Annu Rev Microbiol. 2001; 55(1):357-80.

475 - De Cock, M., Virgilio, M., Vandamme, P., Bourtzis, K., De Meyer, M., \& Willems, A. 476 (2020). Comparative microbiomics of tephritid frugivorous pests (Diptera: Tephritidae) from the field: A tale of high variability across and within species. Front Microbiol, 1890. 
- Dunn OJ. Multiple comparisons among means. J Am Stat Assoc. 1961; 56(293):52-64.

479 - Folch PL, Bisschops MM, Weusthuis RA. Metabolic energy conservation for fermentative $480 \quad$ product formation. Microb Biotechnol. 2020.

481 - Glassing A, Dowd SE, Galandiuk S, Davis B, Chiodini RJ. Inherent bacterial DNA contamination of extraction and sequencing reagents may affect interpretation of microbiota in low bacterial biomass samples. Gut pathog. 2016; 8(1):1-2.

- Goodhue RE, Bolda M, Farnsworth D, Williams JC, Zalom FG. Spotted wing drosophila infestation of California strawberries and raspberries: economic analysis of potential revenue losses and control costs. Pest Manag Sci. 2011; 67(11):1396-402.

- Gurung K, Wertheim B, Falcao Salles J. The microbiome of pest insects: it is not just bacteria. Entomol Exp Appl. 2019; 167(3):156-70.

- Hamby KA, Swett CL. Elucidating symbioses between Drosophila suzukii and fungal communities for improved insect and disease management in raspberry production. North American Bramble Growers Research Foundation Funding. 2015.

- Ioriatti C, Guzzon R, Anfora G, Ghidoni F, Mazzoni V, Villegas TR, Dalton DT, Walton VM. Drosophila suzukii (Diptera: Drosophilidae) contributes to the development of sour rot in grape. J Econ Entomol. 2018; 111(1):283-92.

- Jackson DA. PROTEST: a PROcrustean randomization TEST of community environment concordance. Ecoscience. 1995; 2(3):297-303.

- Johnston PR, Rolff J. Host and symbiont jointly control gut microbiota during complete metamorphosis. PLoS Pathog. 2015; 11(11):e1005246.

499 - Jones AG, Mason CJ, Felton GW, Hoover K. Host plant and population source drive 500 diversity of microbial gut communities in two polyphagous insects. Sci Rep. 2019; 9(1):1- 
- Kembel SW, Cowan PD, Helmus MR, Cornwell WK, Morlon H, Ackerly DD, Blomberg SP, Webb CO. Picante: R tools for integrating phylogenies and ecology. Bioinformatics. $2010 ; 26(11): 1463-4$.

- Kruskal WH, Wallis WA. Use of ranks in one-criterion variance analysis.J Am Stat Assoc.1952; 47(260):583-621.

- Kucuk RA. Bacterial Diversity of the Gut of Cotinis nitida. (2019).

- Kudo R, Masuya H, Endoh R, Kikuchi T, Ikeda H. Gut bacterial and fungal communities in ground-dwelling beetles are associated with host food habit and habitat. ISME J. $2019 ; 13(3): 676-85$.

- $\quad$ Lahti L, Shetty S. Tools for microbiome analysis in R. Version 1.1. 10013. 2017

- Leff JW, Fierer N. Bacterial communities associated with the surfaces of fresh fruits and $513 \quad$ vegetables. PloS One. 2013; 8(3):e59310.

514 - Lewis MT, Hamby KA. Differential impacts of yeasts on feeding behavior and 515 development in larval Drosophila suzukii (Diptera: Drosophilidae). Sci Rep. 2019; 9(1):15162

517 - Lu, M., Hulcr, J., \& Sun, J. (2016). The role of symbiotic microbes in insect 518 invasions. Annual Review of Ecology, Evolution, and Systematics, 47, 487-505.

519 - Lozupone C, Knight R. UniFrac: a new phylogenetic method for comparing microbial $520 \quad$ communities. Appl Environ Microbiol. 2005; 71(12):8228-35.

521 - Majumder, R., Sutcliffe, B., Taylor, P. W., \& Chapman, T. A. (2019). Next-Generation 522 Sequencing reveals relationship between the larval microbiome and food substrate in 523 the polyphagous Queensland fruit fly. Scientific reports, 9(1), 1-12.

524 - Malacrinò A, Campolo O, Medina RF, Palmeri V. Instar-and host-associated 525 differentiation of bacterial communities in the Mediterranean fruit fly Ceratitis capitata. PloS One. 2018; 13(3):e0194131. 
- Marín-Cevada V, Caballero-Mellado J, Bustillos-Cristales R, Muñoz-Rojas J, Mascarúa-Esparza MA, Castañeda-Lucio M, López-Reyes L, Martínez-Aguilar L, Fuentes-Ramírez LE. Tatumella ptyseos, an unrevealed causative agent of pink disease in pineapple. J Phytopathol. 2010; 158(2):93-9.

- Martin M. Cutadapt removes adapter sequences from high-throughput sequencing reads. EMBnet J. 2011; 17(1):10-2.

- Martinson, V. G., Douglas, A. E., \& Jaenike, J. (2017). Community structure of the gut microbiota in sympatric species of wild Drosophila. Ecology Letters, 20(5), 629-639.

- Martinez-Sañudo I, Simonato M, Squartini A, Mori N, Marri L, Mazzon L. Metagenomic analysis reveals changes of the Drosophila suzukii microbiota in the newly colonized regions. Insect sci. 2018; 25(5):833-46.

- Matthies C, Springer N, Ludwig W, Schink B. Pelospora glutarica gen. nov., sp. nov., a glutarate-fermenting, strictly anaerobic, spore-forming bacterium. Int $\mathrm{J}$ Syst Evol Microbiol. 2000; 50(2):645-8. and graphics of microbiome census data. PLoS One. 2013; 8(4), e61217.

$544 \quad$ inadmissible. PLoS Comput Biol. 2014; 10(4):e1003531.

545 - Morotomi M, Nagai F, Watanabe Y, Tanaka R. Succinatimonas hippei gen. nov., sp. 546 nov., isolated from human faeces.Int J Syst Evol Microbiol. 2010; 60(8):1788-93.

547 - Oksanen J. Vegan: an introduction to ordination. URL http://cran. r-project. $548 \quad$ org/web/packages/vegan/vignettes/introvegan. pdf. 2015; 8:19.

549 - R Core Team. R: A language and environment for statistical computing. R Foundation for Statistical Computing, Vienna, Austria. 2020; URL R-project.org/. 
- Rassati D, Marini L, Malacrinò A. Acquisition of fungi from the environment modifies ambrosia beetle mycobiome during invasion. PeerJ. 2019; 7:e8103.

- Raza MF, Yao Z, Bai S, Cai Z, Zhang H. Tephritidae fruit fly gut microbiome diversity, function and potential for applications. Bull. Entomol. Res. 2020; 110:423-347.

- Riley AB, Kim D, Hansen AK. Genome sequence of "Candidatus Carsonella ruddii" strain BC, a nutritional endosymbiont of Bactericera cockerelli. Genome Announc. 2017; 5(17).

- Rivas-Marín E, Devos DP. The Paradigms They Are a-Changin': past, present and future of PVC bacteria research. Antonie Van Leeuwenhoek. 2018; 111(6):785-99.

- Rivers AR, Weber KC, Gardner TG et al. ITSxpress: Software to rapidly trim internally transcribed spacer sequences with quality scores for marker gene analysis. F1000Res. 2018; 7:1418 (doi: 10.12688/f1000research.15704.1) BioMed Res. Int. 2013; 2013.

- Rota-Stabelli O, Blaxter M, Anfora G. Drosophila suzukii. Current Biology. 2013; 23(1):R8-9. environments: larval performance, foraging decisions, and adult oviposition choices in

$571 \quad$ Drosophila suzukii. BMC Ecol. 2017; 17(1):1-3.

572 - Solomon, G. M., Dodangoda, H., McCarthy-Walker, T., Ntim-Gyakari, R., \& Newell, P. D. 573 (2019). The microbiota of Drosophila suzukii influences the larval development of $574 \quad$ Drosophila melanogaster. PeerJ, 7, e8097. 
- Stackebrandt ER, Hespell R. The family succinivibrionaceae. The prokaryotes. Springer, Berlin, Heidelberg. 2006; 3:419-29.

- Starmer WT, Fogleman JC. Coadaptation of Drosophila and yeasts in their natural habitat. J Chem Ecol. 1986;12(5):1037-55

- Sudarshan A. Shetty, \& Leo Lahti. (2020, October). microbiomeutilities: Utilities for Microbiome Analytics.

- Thao ML, Moran NA, Abbot P, Brennan EB, Burckhardt DH, Baumann P. Cospeciation of psyllids and their primary prokaryotic endosymbionts. Appl Environ Microbiol. 2000; 66(7):2898-905.

- Tissot M, Stocker RF. Metamorphosis in Drosophila and other insects: the fate of neurons throughout the stages.Prog. Neurobiol. 2000; 62(1):89-111.

- Vacchini, V., Gonella, E., Crotti, E., Prosdocimi, E.M., Mazzetto, F., Chouaia, B., Callegari, M., Mapelli, F., Mandrioli, M., Alma, A. and Daffonchio, D., (2017). Bacterial diversity shift determined by different diets in the gut of the spotted wing fly Drosophila suzukii is primarily reflected on acetic acid bacteria. Environmental microbiology reports, 9(2), 91-103.

- van Veelen HP, Salles JF, Matson KD, van der Velde M, Tieleman BI. Microbial environment shapes immune function and cloacal microbiota dynamics in zebra finches Taeniopygia guttata. Animal Microbiome. 2020; 2:1-7. SD, Zalom FG. Drosophila suzukii (Diptera: Drosophilidae): invasive pest of ripening soft fruit expanding its geographic range and damage potential. J Integr Pest Manag. 2011; 2(1):G1-7. 
- Wang, Q, G. M. Garrity, J. M. Tiedje, and J. R. Cole. 2007. Naïve Bayesian Classifier for Rapid Assignment of rRNA Sequences into the New Bacterial Taxonomy. Appl Environ Microbiol. 73(16):5261-7.

- Whitehead SR, Bowers MD. Chemical ecology of fruit defence: synergistic and antagonistic interactions among amides from Piper. Funct Ecol. 2014; 28(5):1094-106. (2019). Similar shift patterns in gut bacterial and fungal communities across the life stages of Bactrocera minax larvae from two field populations. Frontiers in Microbiology, 10, 2262.

614 Table [also attached as separate word file]

615 Table 1. Details of the infested fruit collections (these fruits and the flies that emerged

616 from them were used for microbiome characterization)

\begin{tabular}{|l|l|l|l|l|l|}
\hline Name & Location co- & Type of & Code by fruit & Fruit type & Whether \\
\hline
\end{tabular}




\begin{tabular}{|c|c|c|c|c|c|}
\hline & ordinates & $\begin{array}{l}\text { sampling } \\
\text { site }\end{array}$ & source & & $\begin{array}{l}\text { flies were } \\
\text { lab-reared }\end{array}$ \\
\hline \multirow[t]{3}{*}{ Randwijk } & \multirow[t]{3}{*}{$\begin{array}{l}\text { N5 } 1^{\circ} 56.953^{\prime} \\
E 005^{\circ} 43.041^{\prime}\end{array}$} & Orchard & Cherry_Rw_1 & Cherry & Yes \\
\hline & & Orchard & Cherry_Rw_2 & Cherry & No \\
\hline & & Orchard & Cherry_Rw_3 & Cherry & No \\
\hline \multirow[t]{2}{*}{ Winssen } & \multirow[t]{2}{*}{$\begin{array}{l}\text { N51 } 53.234^{\prime} \\
\text { E00541.372' }\end{array}$} & Orchard & Cherry_Wn_4, & Cherry & No \\
\hline & & Orchard & Raspberry_Wn & Raspberry & No \\
\hline \multirow[t]{3}{*}{ Dirksland } & \multirow[t]{3}{*}{$\begin{array}{l}\text { N51 }{ }^{\circ} 44.63^{\prime} \\
\text { E45.48' }\end{array}$} & Orchard & Raspberry_DI, & Raspberry & No \\
\hline & & Orchard & Strawberry_DI, & Strawberry & Yes \\
\hline & & Orchard & Blackberry_DI & Blackberry & No \\
\hline
\end{tabular}




\begin{tabular}{|l|l|l|l|l|l|}
\hline Hoogeveen & $\begin{array}{l}\text { N52 }{ }^{\circ} 44.61^{\prime} \\
\text { E6 } 6^{\circ} 32.66\end{array}$ & Farm field & Elderberry_Hv & Elderberry & Yes \\
\hline
\end{tabular}

$617{ }^{1}$ Each fly replicate corresponds to a pool of 5 adult flies that emerged from the same individual

618 fruit; each fruit replicate corresponds to approximately $1 \mathrm{~g}$ of fruit.

$619{ }^{2}$ Cherries from location Randwijk belonged to three different (and unknown) cultivars

620 Table 2. Procrustes analysis indicating the association between the bacterial and fungal

621 communities from wild flies and their corresponding fruit samples as well as between lab

622 flies and wild flies.

\begin{tabular}{|l|l|l|l|}
\hline Environment & Organism & M 2 & p-value \\
\hline Wild fly-fruit & bacteria & 0.934 & 0.197 \\
\hline Wild fly-fruit & fungi & 0.554 & 0.001 \\
\hline Lab-wild fly & bacteria & 0.529 & 0.003 \\
\hline
\end{tabular}


Figure legends

628 Figure 1. Alpha diversity (number of observed ASVs) of bacterial (a) and fungal (b) 629 communities associated with fruits and wild D. suzukii flies, arranged by fruit type and collection 630 site. The richness of both bacterial and fungal communities across fruits and the emerging wild 631 flies differed significantly ( $p$-values $<0.1$ and $<0.05$, respectively).

Figure 2. Beta diversity of bacterial ( $a$ and $b$ ) and fungal ( $c$ and $d)$ communities associated with

634 fruits (triangles) and wild D. suzukii (dots). a: PCoA of weighted UniFrac distance of bacterial 635 communities differed significantly between fruits and flies ( $p$-value=0.001). b: Individual facets

636 of PCoA using weighted UniFrac for every fruit type in every collection site. c: PCoA of Bray 637 Curtis dissimilarity index for fungal communities, which differed significantly between fruits and 638 flies ( $p$-value=0.021). d: Individual facets of PCoA using Bray Curtis by fruit type in every 639 collection site.

641 Figure 3. Alpha and beta diversity (of bacterial communities across wild and lab-reared flies.

642 Alpha diversity (observed ASVs) of a) did not differ significantly between wild and lab-reared 643 flies ( $p$-value=0.40). Beta diversity $c$ ) based on Weighted UniFrac distances for bacterial 644 communities differs significantly between the lab-reared and wild flies ( $p$-value=0.001) 
bioRxiv preprint doi: https://doi.org/101101/2022 02.28 482158; this version posted March 2, 2022. The copyright holder for this preprint (which was not certified by peer review) is the author/funder, who has granted bioRxiv a license to display the preprint in perpetuity. It is made available under aCC-BY-NC-ND 4.0 International license.

646 Figure 4. Top 10 abundant bacteria across wild flies and their source fruits (a). Four core

647 bacteria were observed across the wild flies (b). Top 10 abundant bacteria across lab-reared

648 flies and wild flies, which also comprised three of the core bacteria (c). Top 10 abundant fungi

649 across wild flies and their corresponding fruits $(d)$.

650

651 Data availability statement:

652 Additional files and data available in the link: https://figshare.com/s/4eebffffaab656b540f8 
Main figures 


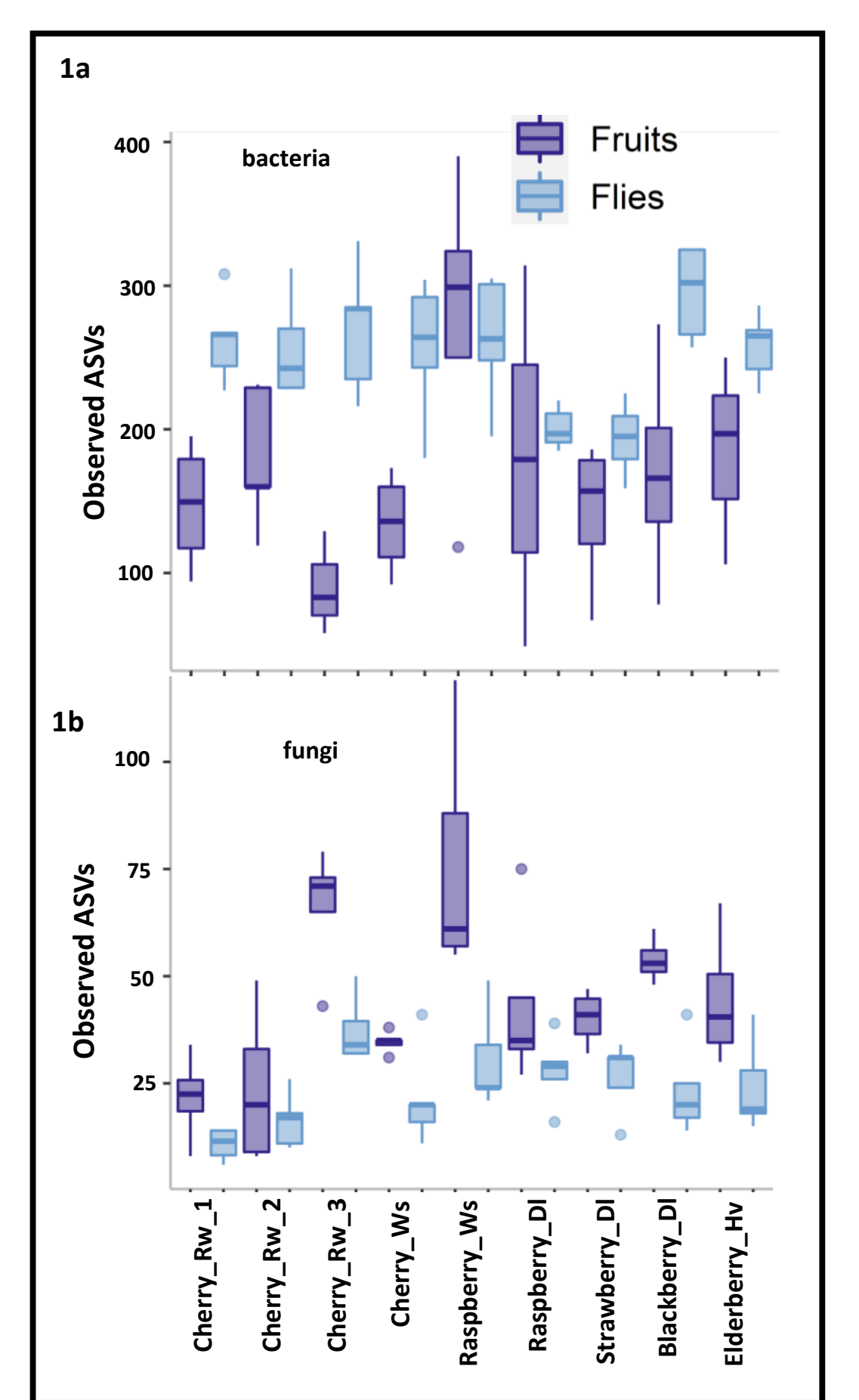




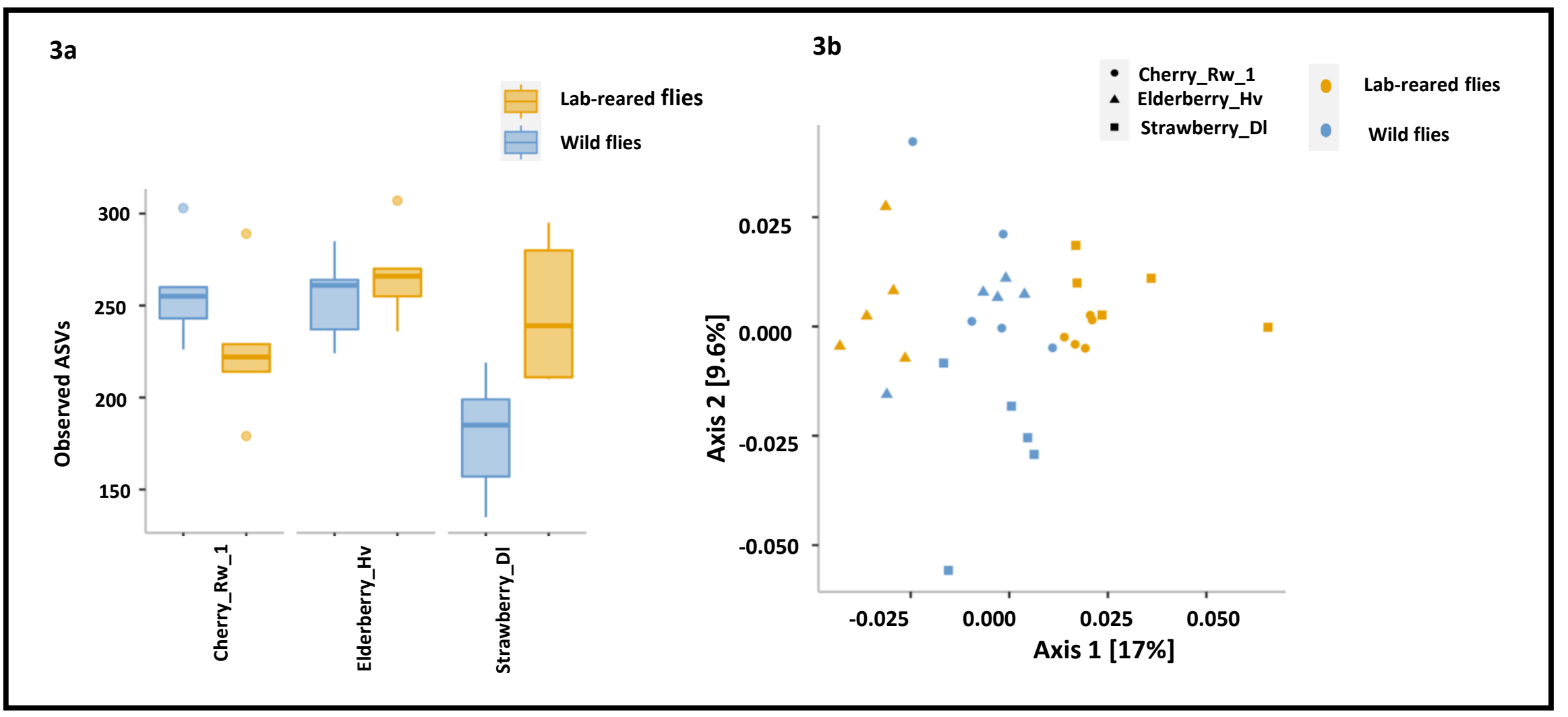


Wild flies

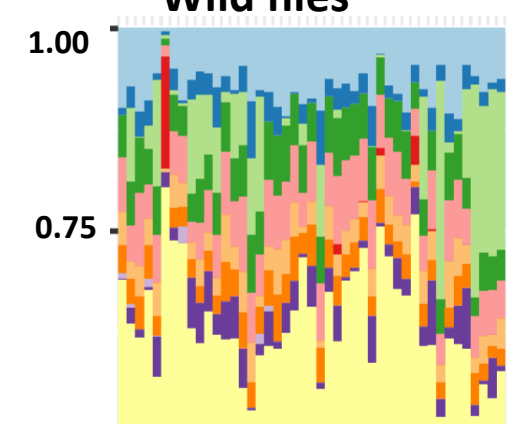

0.50

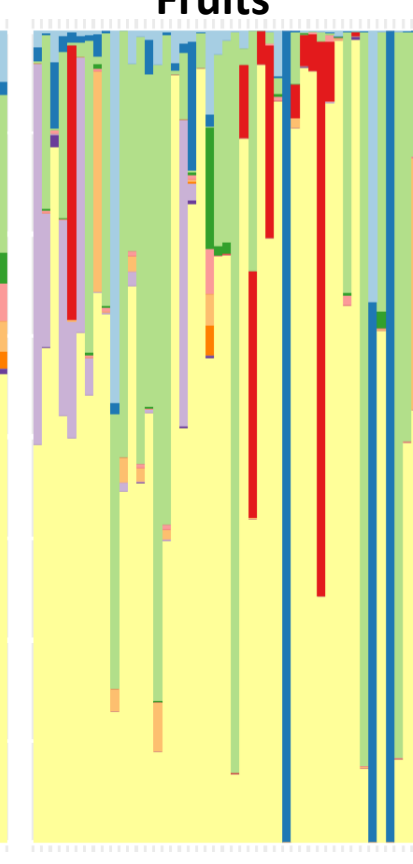

0.25 -

0.00 .

\section{Bacteria}

g_Candidatus Carsonella

g_Candidatus Scalindua

g_Gluconobacter

g_Minicystis

g_Omnitrophica_genera_incertae_sedis

g_Pantoea

g_Pelospora

g_Stella

g_Tatumella

g_Tepidisphaera

Other

$4 c$

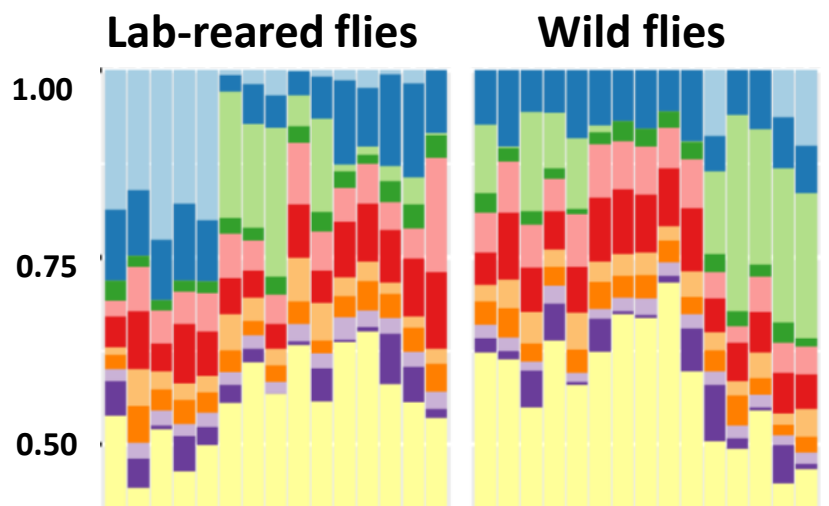

0.25

\section{Core bacteria in wild flies}

0.20

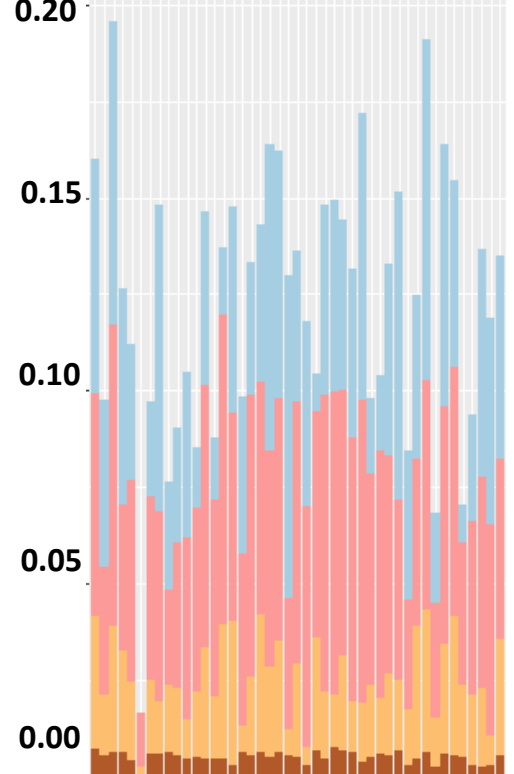

\section{Bacteria}

g_Candidatus Carsonella

g_Omnitrophica_genera_incertae_sedis

g_Pelospora

g_Succinatimonas

4d

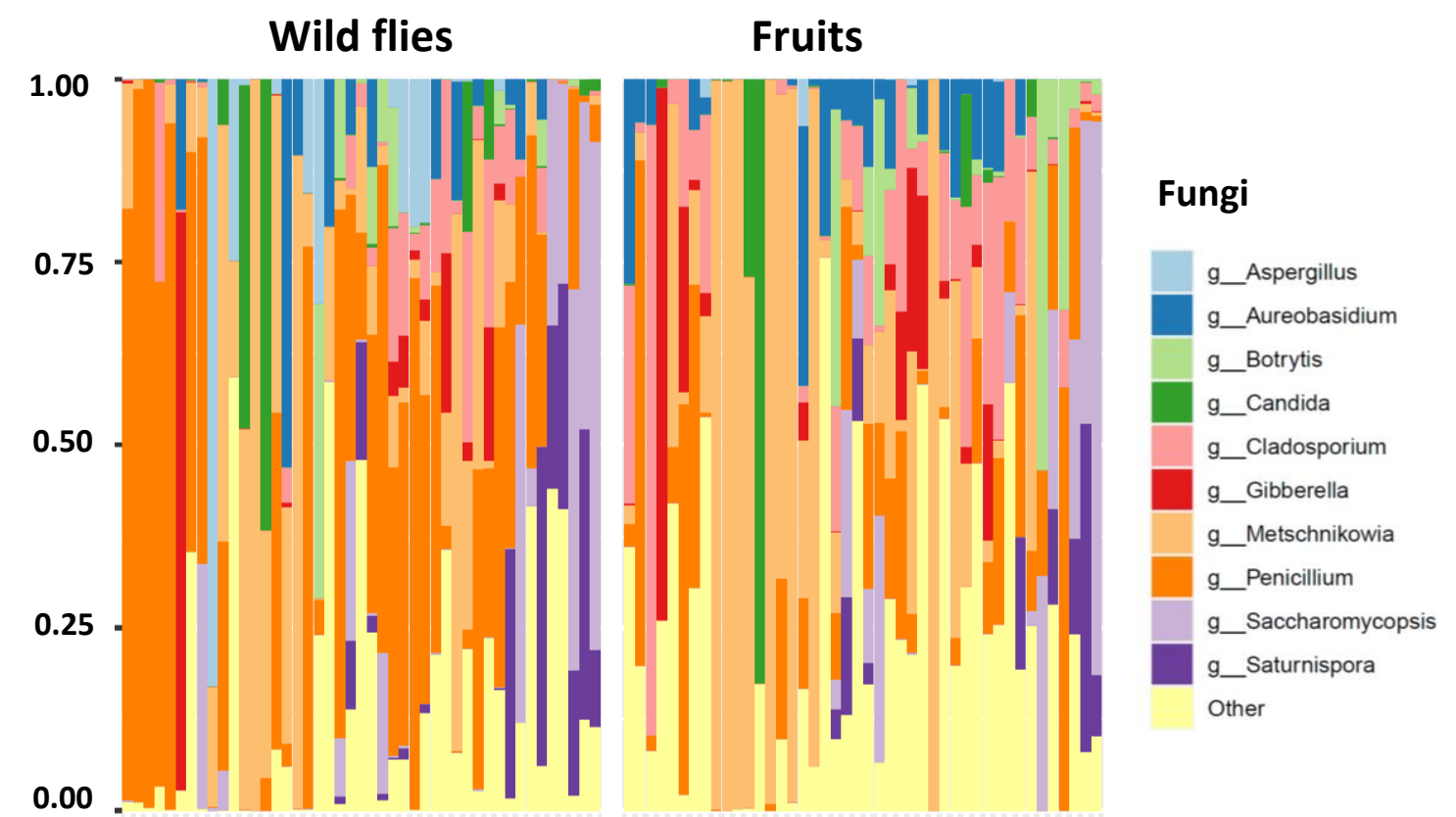

